\title{
Integración de la tenencia y la gobernanza en las evaluaciones de oportunidades de restauración del paisaje forestal
}

\author{
Rebecca McLain ${ }^{a}$, Steven Lawry ${ }^{b}$ Manuel R. Guariguata ${ }^{c}$ y James Reed ${ }^{c}$
}

\section{Mensajes clave}

- Varios países han adoptado la Metodología de Evaluación de Oportunidades de Restauración (ROAM de sus siglas en inglés) como guía para desarrollar estrategias de restauración de escala nacional y subnacional.

- Este estudio analiza informes ROAM de ocho países para determinar en qué medida fueron incluidos los aspectos de tenencia y de gobernanza relacionados.

- Aunque todos los informes reportan que la carencia de derechos o la debilidad de estos obstaculiza los esfuerzos para ampliar la escala de la restauración del paisaje forestal (RPF), en ninguno de los informes se brindó una descripción robusta de los derechos y responsabilidades de individuos o comunidades respecto a árboles, bosques o tierra, conforme al derecho consuetudinario o estatutario.

- Proponemos como diagnóstico un marco de actualización de derechos que provea fundamentos sólidos para identificar reformas necesarias de políticas para abordar los obstáculos que tienen que ver con derechos en la implementación de la RPF.

- Las iniciativas de RPF informadas por una sólida evaluación de derechos de tenencia habrá de mejorar la probabilidad de alcanzar sus objetivos de incrementar tanto la funcionalidad ecológica como el bienestar humano.

\section{Por qué el tema de tenencia es importante en la restauración del paisaje forestal (RPF)}

Las guías para la restauración del paisaje forestal (RPF) (p. ej. Mansourian 2017; Stanturf et al. 2017) subrayan la importancia de mejorar los derechos de tenencia de la comunidad y de los pequeños propietarios, la seguridad de la tenencia y los sistemas de gobernanza de la tierra para alcanzar objetivos de índole ambiental y social. Es probable que dado que tanto la tenencia como la gobernanza afectan los costos de RPF y la distribución de beneficios, estos aspectos influyan en las decisiones sobre tenencia y uso de la tierra respecto a la inversión en prácticas de RPF (Cronkleton et al. 2017). La Metodología de Evaluación de Oportunidades de Restauración (ROAM) desarrollada por la Unión Internacional para la Conservación de la Naturaleza (UICN) y el Instituto de

a CIFOR, Bogor, Indonesia; Instituto para Soluciones Sostenibles, Universidad Estatal de Portland

b CIFOR, Bogor, Indonesia

c CIFOR, Lima, Perú

d CIFOR, Bogor, Indonesia; Profesor visitante, Instituto de Investigación en Conservación de la Universidad de Cambridge, Edificio David Attenborough, Cambridge, CB2 3QZ, Reino Unido
Recursos Mundiales (WRI de sus siglas en inglés) es un proceso diseñado para informar estrategias de planeamiento de RPF nacionales y subnacionales. Las guías se incluyen en un manual (UICN y WRI 2014) y en la publicación del WRI titulada Diagnóstico de la Restauración (WRI 2015), que estimula a los profesionales a incorporar los aspectos de tenencia y gobernanza que tienen la probabilidad de afectar aceptación de la RPF. Evaluamos ocho informes ROAM (ver la tabla 1) con el fin de analizar de qué forma y si la tenencia y gobernanza se incorporaron en los procesos de evaluación de oportunidades de RPF. El análisis se basó en tres preguntas:

- ¿Cómo y dónde los informes ROAM consideran los derechos de tenencia y los aspectos de gobernanza asociados?

- ¿Cuáles fueron los desafíos identificados relacionados con derechos de tenencia y gobernanza?

- ¿Qué soluciones se proponen para abordar los posibles desafíos de tenencia y gobernanza que afectan la implementación de la RPF?

Con base en nuestros resultados, brindamos recomendaciones para un diagnóstico de la tenencia de forma que se pueda complementar con evaluaciones ROAM u otras evaluaciones de la RPF, así como una agenda orientada a mejorar la incorporación de los derechos de tenencia en la planeación y programación de la RPF. 


\section{Métodos}

Identificamos informes ROAM mediante el motor de búsqueda Google y examinando los sitios web relacionados con la RPF (p. ej. UICN s.f., 2018). La búsqueda se limitó a los informes publicados entre los años 2011 y 2017; se tomó el año 2011 como la fecha a partir de la cual se dirigieron los primeros procesos ROAM. Las palabras clave incluyeron: restauración forestal, restauración del paisaje forestal, evaluación de oportunidades de restauración, ROAM, diagnóstico de restauración forestal, incluyendo las traducciones de estas palabras al francés, inglés y portugués como se emplean en la serie de manuales de ROAM (UICN 2019). Ubicamos seis informes ROAM nacionales y subnacionales (tabla 1), seis en África y dos en América Latina. Tres de los informes correspondieron a actividades piloto (Ghana, Guatemala y Ruanda) y culminaron antes de la publicación del manual ROAM. Dado que la metodología ROAM todavía está en sus etapas iniciales de desarrollo, es probable que sean incorporados nuevos componentes en los procesos en la medida en que las personas a cargo acumulen experiencia al darle uso. Con el fin de desarrollar criterios de evaluación de la inclusión de aspectos de derechos de tenencia y seguridad de tenencia en los informes ROAM, combinamos visiones de la teoría y de la investigación empírica en cuanto los derechos de propiedad y las inversiones en conservación, así como los factores de éxito descritos en las guías ROAM publicadas. Los criterios de evaluación incluyeron derechos de tenencia, seguridad de tenencia, capacidad de dar cumplimiento, compromiso de la comunidad, consistencia de políticas y del marco legal, y enlaces de múltiples escalas o múltiples sectores (véase la tabla 2).

\section{Visión general del proceso ROAM}

A pesar de que no existe un formato ROAM estándar, los procesos subyacentes de los informes ROAM siguieron un patrón muy similar.

Cada proceso comenzó con un taller de múltiples partes interesadas para identificar potenciales intervenciones de RPF y criterios de evaluación. A continuación, un grupo de trabajo desarrolló mapas señalando dónde sería factible cada tipo de intervención. En visitas de campo y talleres subnacionales se obtuvieron aportes de las partes interesadas subnacionales y locales. Muchos países incorporaron uno o más de los siguientes análisis en sus evaluaciones: un análisis de costo-beneficio de las actividades de restauración, un análisis del valor del secuestro de carbono, una evaluación de financiamiento y abastecimiento de restauración, y un análisis político e institucional. Luego de haber terminado los análisis, normalmente se llevó a cabo un taller nacional para brindar a un grupo ampliado de partes interesadas la oportunidad de discutir, validar y refinar los resultados. A continuación el equipo principal produjo un informe final en el que se resumen los resultados y brindan recomendaciones para los planes o estrategias de RPF nacionales o subnacionales.

\section{Manejo del aspecto de tenencia en los informes ROAM}

Los participantes en la metodología ROAM tienen un margen de maniobra considerable en la forma en que interpretan y aplican
Tabla 1. Informes de Metodología de Evaluación de Oportunidades de Restauración (ROAM) incluidos en este estudio.

\begin{tabular}{lc}
\hline País (escala de la evaluación) & $\begin{array}{c}\text { Fecha de } \\
\text { publicación }\end{array}$ \\
\hline Etiopía (Estado de Amhara) & 2017 \\
Ghana* (nacional) & 2011 \\
Costa de Marfil (nacional) & 2016 \\
Malawi (nacional) & 2017 \\
Ruanda* (nacional) & 2014 \\
Uganda (nacional) & 2016 \\
Brasil (Estado de Pará) & 2017 \\
Guatemala* (nacional) & 2014 \\
\hline
\end{tabular}

* Ghana, Guatemala y Ruanda fueron experiencias piloto

Tabla 2. Marco empleado para evaluar la consideración de aspectos de tenencia y de gobernanza de la tierra en los informes de Metodología de Evaluación de Oportunidades de Restauración (ROAM).

\begin{tabular}{ll}
\hline $\begin{array}{l}\text { Dimensión } \\
\text { de Tenencia/ } \\
\text { gobernanza de la } \\
\text { tierra }\end{array}$ & $\begin{array}{l}\text { Indicador de cobertura de los aspectos de } \\
\text { tenencia y gobernanza de la tierra }\end{array}$ \\
\hline $\begin{array}{l}\text { Derechos de } \\
\text { tenencia }\end{array}$ & $\begin{array}{l}\text { Describe los derechos y responsabilidades } \\
\text { (estatutarios y consuetudinarios) de los } \\
\text { propietarios de tierras con relación a árboles, } \\
\text { bosques, tierra y carbono, incluyendo los } \\
\text { derechos de mujeres, minorías y grupos en } \\
\text { desventaja }\end{array}$
\end{tabular}

Describe forestería comunitaria o mecanismos de cogestión

Seguridad de tenencia

Capacidad de cumplimiento

Participación de la comunidad

Consistencia de políticas y del marco legal

Vínculos a múltiples escalas y entre múltiples sectores
States whether land or resource tensions or conflicts are present and describes the nature of those tensions or conflicts

Establece si se presentanya sea tensiones sobre tierras, recursos o conflictos y describe la naturaleza de tales tensiones o conflictos

Describe la capacidad de cumplimiento (en la comunidad y el gobierno)

Describe el nivel de participación de la comunidad en la toma de decisiones de la RPF

Describe la presencia (o ausencia) de inconsistencias en políticas y marcos legales que probablemente afecten las intervenciones de RPF

Describe si existen actores a diferentes escalas y en diferentes sectores que coordinan el diseño de políticas, la planeación y la implementación de un programa relacionado con la RPF, y cómo lo hacen 


\title{
Tabla 3. Sistema de calificación para evaluar la medida en que se consideró la tenencia y la gobernanza en informes de Metodología de Evaluación de Oportunidades de Restauración (ROAM).
}

\author{
Calificación para la cobertura Criterio de calificación \\ sobre tenencia y gobernanza \\ de la tierra
}

Cobertura muy limitada

Breve mención de problemas de tenencia en una o más secciones con poco detalle; no incluye una lista o discusión de leyes o políticas clave que puedan afectar los incentivos de tenencia por participar en la RPF; no describe derechos estatutarios y responsabilidades respecto a árboles o tierra; no describe derechos y responsabilidades consuetudinarios respecto a árboles o tierras; la información es poco específica en cuanto a identificar reformas políticas apropiadas o medidas de acompañamiento

\begin{tabular}{ll}
\hline Cobertura limitada & $\begin{array}{l}\text { Hace mención de temas de tenencia en una o más secciones pero con poco detalle; hace un listado } \\
\text { de políticas o leyes clave que afectan los incentivos de tenencia para la participación en la RPF; brinda } \\
\text { una idea de cuáles grupos tienen derechos de tenencia débiles o nulos; ofrece alguna información } \\
\text { sobre derechos y responsabilidades respecto a árboles o tierras bajo ya sea normas estatutarias o } \\
\text { consuetudinarias; la información sobre tenencia no es específica en la identificación de apropiadas } \\
\text { reformas de políticas y medidas de acompañamiento }\end{array}$ \\
\hline $\begin{array}{l}\text { Cobertura moderada pero } \\
\text { restringida }\end{array}$ & $\begin{array}{l}\text { Ofrece una discusión considerable sobre temas de tenencia en una o más seccione; hace un listado } \\
\text { o discute leyes o políticas clave que afectan incentivos de tenencia para la participación en la RPF; } \\
\text { brinda una idea de los grupos con derechos de tenencia débiles o nulos; brinda alguna información } \\
\text { sobre derechos y responsabilidades respecto a árboles y tierras bajo leyes estatutarias o normas } \\
\text { consuetudinarias, o bajo ambas: la información sobre uno o más temas clave de tenencia es lo } \\
\text { suficientemente específica de manera que permite identificar reformas apropiadas de políticas y medidas } \\
\text { de acompañamiento }\end{array}$ \\
\hline
\end{tabular}

utilizando el sistema de clasificación descrito en la tabla 3. En la tabla 4 se resume la cobertura general de tenencia y gobernanza de la tierra en los ocho informes. Como sucede en todo registro escrito sobre algún hecho, los informes proporcionan solo una imagen parcial de lo que realmente ocurrió durante las evaluaciones. Análisis geoespacial: Todos los informes incluyeron análisis geoespacial mediante el cual se identificaron sitios de RPF. Los límites de áreas protegidas fueron las únicas capas de datos de tenencia incluidas en todos los informes menos uno. El informe del estado de Pará en Brasil también incorporó capas de datos de Reserva Legal y Áreas de Protección Permanente que han sido registradas en el Registro Ambiental Rural.

Análisis de costo-beneficio: En siete informes se incorporaron análisis de intervenciones de RPF1. Cinco informes utilizaron un enfoque cuantitativo en el que se suponía que los individuos o las comunidades que plantaban o protegían árboles tendrían derecho a cosechar y vender los árboles o sus productos. Este supuesto no tiene en cuenta las situaciones en que los individuos o las comunidades no tienen estos derechos. Además, los análisis no parecen haber incluido los costos de transacción asociados a la obtención de permiso de cosecha o de transporte. Los informes de Ghana y de Costa de Marfil emplearon un enfoque cualitativo en sus análisis de costo-beneficio. En ambos informes se incluyó una lista de asuntos que necesitan resolverse con el fin de implementar la RPF en diferentes categorías de tierras; los elementos en la lista se consideran costos. También se desarrolló una lista de beneficios

1 En 2018, luego de culminar nuestro estudio, se publicó un documento para Guatemala incluyendo un análisis de costo-beneficio de intervenciones de RPF. La referencia es: J Colomer, A Imbach, L Raes, U Parrilla, F Reinhard, M Fernandez y M Allemant. 2018. Value for Money: Guatemala's Forest Landscape Restoration. Gland, Suiza: UICN espera-dos. Se incluyeron en los costos los aspectos de tenencia y gobernanza, como la carencia de derechos de los agricultores migrantes sobre los árboles y un deficiente cumplimiento de las restricciones a la tumba de árboles.

Financiamiento para la restauración: Los informes de Ruanda, Malawi, Pará y Guatemala trataron el tema de opciones de financiamiento para la restauración. Se enfatizó en la descripción de fuentes externas de financiamiento potencial y existente para las comunidades. Los informes de Ruanda, Pará y Malawi identificaron el incentivar a los pequeños agricultores a invertir en la restauración de sus propiedades como algo esencial, con la asistencia a las pequeñas y medianas empresas como el mecanismo de apoyo clave. El informe de Guatemala también hizo hincapié en el papel clave que han desempeñado los derechos de concesión de bosques comunitarios para incentivar a los miembros de la comunidad a extraer madera de manera sostenible en áreas protegidas. En el informe de Malawi se exploró el financiamiento con base comunitaria como una opción, con la mención de fondos provenientes de los comités de manejo forestal del poblado como una posible fuente de financiamiento.

Análisis de valor de carbono: Los informes de Ruanda y de Ghana brindaron breves discusiones sobre derechos de carbono, en tanto que en los demás informes estuvieron ausentes los temas de derechos de carbono.

Análisis de políticas e instituciones: En todos los informes se incluyó un análisis de políticas e instituciones. Sin embargo, el grado en que los detalles sobre la tenencia y la gobernanza fueron proporcionados variaba enormemente. Algunos informes apenas incluyeron una lista de leyes relacionadas con la RPF y otros describieron las disposiciones clave de las leyes relevantes. Algunos informes incluyeron solo declaraciones resumidas de temas de tenencia, como "tenencia 
Tabla 4. Cobertura en temas de tenencia en informes sobre Metología de Evaluación de Oportunidades de Restauración (ROAM).

\begin{tabular}{|c|c|c|c|c|c|c|}
\hline País & $\begin{array}{l}\text { Evaluación general } \\
\text { de la cobertura de } \\
\text { temas de aspectos de } \\
\text { tenencia }\end{array}$ & $\begin{array}{l}\text { Datos de } \\
\text { tenencia } \\
\text { incluidos en } \\
\text { el análisis } \\
\text { geoespacial }\end{array}$ & $\begin{array}{l}\text { Tipo de análisis } \\
\text { de costo- } \\
\text { beneficio }\end{array}$ & $\begin{array}{l}\text { Se incluyó } \\
\text { un análisis } \\
\text { financiero de la } \\
\text { restauración }\end{array}$ & $\begin{array}{l}\text { Se incluyó un } \\
\text { análisis de valores } \\
\text { de carbono }\end{array}$ & $\begin{array}{l}\text { Tipo de } \\
\text { análisis de } \\
\text { políticas e } \\
\text { instituciones }\end{array}$ \\
\hline Ruanda & Cobertura muy limitada & $\begin{array}{l}\text { Tipo de análisis } \\
\text { de políticas e } \\
\text { instituciones }\end{array}$ & Quantitative & Sí & $\begin{array}{l}\text { Describe brevemente } \\
\text { los derechos de } \\
\text { carbono }\end{array}$ & $\begin{array}{l}\text { Restarauración } \\
\text { Diagnóstico }\end{array}$ \\
\hline Uganda & Cobertura limitada & $\begin{array}{l}\text { Park and reserve } \\
\text { locations }\end{array}$ & Cuantitativo & No & $\begin{array}{l}\text { No describe derechos } \\
\text { de carbono }\end{array}$ & $\begin{array}{l}\text { Restarauración } \\
\text { Diagnóstico }\end{array}$ \\
\hline Malawi & $\begin{array}{l}\text { Cobertura moderada } \\
\text { pero restringida }\end{array}$ & $\begin{array}{l}\text { No describe } \\
\text { derechos de } \\
\text { carbono }\end{array}$ & $\begin{array}{l}\text { Restarauración } \\
\text { Diagnóstico }\end{array}$ & Sí & $\begin{array}{l}\text { Incluido en un análisis } \\
\text { financiero de un } \\
\text { apéndice; no hace } \\
\text { mención a derechos } \\
\text { de carbono }\end{array}$ & $\begin{array}{l}\text { Restarauración } \\
\text { Diagnóstico }\end{array}$ \\
\hline $\begin{array}{l}\text { Etiopía } \\
\text { (Amhara) }\end{array}$ & $\begin{array}{l}\text { Cobertura moderada } \\
\text { pero restringida }\end{array}$ & Áreas protegidas & $\begin{array}{l}\text { Restarauración } \\
\text { Diagnóstico }\end{array}$ & No & $\begin{array}{l}\text { No describe derechos } \\
\text { de carbono }\end{array}$ & $\begin{array}{l}\text { Restarauración } \\
\text { Diagnóstico }\end{array}$ \\
\hline Costa de Marfil & Cobertura limitada & $\begin{array}{l}\text { No se incluyen } \\
\text { variables de } \\
\text { tenencia }\end{array}$ & Cuantitativo & No & $\begin{array}{l}\text { No describe derechos } \\
\text { de carbono }\end{array}$ & $\begin{array}{l}\text { Listado de leyes } \\
\text { y políticas }\end{array}$ \\
\hline Ghana & $\begin{array}{l}\text { Cobertura moderada } \\
\text { pero restringida }\end{array}$ & $\begin{array}{l}\text { Límites de reservas } \\
\text { forestales y parques } \\
\text { nacionales }\end{array}$ & Cuantitativo & No & $\begin{array}{l}\text { Hace un llamado } \\
\text { para la legislación de } \\
\text { derechos de carbono }\end{array}$ & $\begin{array}{l}\text { Discusión } \\
\text { narrativa sobre el } \\
\text { marco político/ } \\
\text { legal }\end{array}$ \\
\hline Brasil (Pará) & $\begin{array}{l}\text { Cobertura moderada } \\
\text { pero restringida }\end{array}$ & $\begin{array}{l}\text { Territorios } \\
\text { indígenas y áreas } \\
\text { de conservación, } \\
\text { parcelas de Registro } \\
\text { Ambiental Rural }\end{array}$ & Cuantitativo & $\begin{array}{l}\text { No como una } \\
\text { sección separada } \\
\text { dentro del } \\
\text { informe pero se } \\
\text { discutió en otras } \\
\text { secciones }\end{array}$ & $\begin{array}{l}\text { No describe derechos } \\
\text { de carbono pero } \\
\text { identifica pagos } \\
\text { por servicios del } \\
\text { ecosistema como } \\
\text { esencial para el éxito } \\
\text { de la RPF }\end{array}$ & $\begin{array}{l}\text { Restoration } \\
\text { Diagnostic }\end{array}$ \\
\hline Guatemala & $\begin{array}{l}\text { Cobertura moderada } \\
\text { pero restringida }\end{array}$ & $\begin{array}{l}\text { Sí, pero el mapa se } \\
\text { publicó como un } \\
\text { documento aparte }\end{array}$ & $\begin{array}{l}\text { Análisis de } \\
\text { costo-beneficio } \\
\text { publicado en } \\
\text { un documento } \\
\text { aparte }\end{array}$ & $\begin{array}{l}\text { No como una } \\
\text { sección separada } \\
\text { dentro del } \\
\text { informe pero se } \\
\text { discutió en otras } \\
\text { secciones }\end{array}$ & $\begin{array}{l}\text { Los derechos de } \\
\text { carbono no se } \\
\text { mencionan en } \\
\text { discusiones sobre } \\
\text { derechos }\end{array}$ & $\begin{array}{l}\text { Discusión } \\
\text { narrativa sobre el } \\
\text { marco político/ } \\
\text { legal }\end{array}$ \\
\hline
\end{tabular}

insegura" o "débil cumplimiento". Otras describieron qué segmentos de la población poseían acceso inseguro a la tierra (es decir, agricultores migrantes en Ghana, mujeres en Malawi y en Uganda), cuáles instituciones carecen de capacidad o de autoridad para hacer cumplir las leyes forestales, y qué leyes eran difíciles de hacer cumplir. En ningún informe se incluyó una descripción sistemática de los derechos y responsabilidades de individuos o comunidades respecto a árboles, bosques o tierras bajo leyes consuetudinarias o estatutarias. No se consideraron derechos de pastores y de usuarios secundarios en ninguno de los informes. Solo se mencionaron diferencias de género en cuanto a derecho a árboles y tierra en los informes de Uganda y Malawi.

A pesar de su limitada cobertura de los derechos de tenencia, los informes ROAM brindaron información útil sobre los desafíos de tenencia que probablemente se presentarán al implementar programas de RPF, así como algunas soluciones a dichos desafíos. Los desafíos más comunes identificados en los informes fueron débil aplicación, limitada participación de la comunidad en la toma de decisiones forestales y la carencia de coordinación entre sectores, actores y escalas. Como solución, los informes de los países africanos enfatizaron en la necesidad de reconocer o devolver los derechos a las autoridades tradicionales y ampliar la participación de la comunidad en la gestión forestal tanto dentro como fuera de las reservas forestales. El registro de tierras en manos de individuos o comunidades, o ambas, se identificó como una solución a la inseguridad de tenencia en todos los informes. Otra solución común planteada fue la expansión de los derechos de cosecha de árboles y otros productos forestales para uso comercial como una forma de aumentar los incentivos para invertir en la RPF. 


\section{Recomendaciones para futuras evaluaciones de RPF}

Ofrecemos dos conjuntos de recomendaciones para futuras ROAM y evaluaciones similares de RPF. Tomando en cuenta que nuestro tamaño de muestra es limitado y que nos concentramos en los informes publicados durante los primeros años de desarrollo de la metodología ROAM, ambas ofertas son provisionales. Un conjunto de recomendaciones describe los elementos de lo que podría incluir un análisis ROAM que considera la tenencia. El otro describe una agenda de RPF que considera la tenencia que se basa en las lecciones de REDD+ y en iniciativas forestales comunitarias.

El manual ROAM y el Diagnóstico de Restauración brindan un punto de partida para la incorporación de los aspectos de tenencia y gobernanza, pero la información resultante del uso de estas herramientas son demasiado generales como para ser de utilidad en la planeación de intervenciones de RPF. Concluimos que un diagnóstico específico para la tenencia, que también incorpora cuestiones de gobernanza y equidad, ofrece una posible solución. Proponemos un modelo de actualización de derechos (véase la figura 1) como la base para un diagnóstico de tenencia el cual le permite identificar áreas geográficas en donde la tenencia podría estimular o desalentar la adopción de intervenciones de RPF a quienes elaboran los planes, así como la identificación del tipo de reformas de tenencia y de reformas de gobernanza que habrán de requerirse para apoyar la restauración a gran escala. Los derechos reales, a los cuales se refieren BendaBeckmann y von Benda-Beckmann (2000) como derechos concretos, son aquellos cuyo titular puede ejercer en la práctica.
El punto de partida del modelo (Elemento A) consiste en derechos en la ley, ya sea estatuaria o consuetudinaria. Estos derechos están típicamente condicionados de varias maneras (Elemento B). Los derechos condicionados están además condicionados por las percepciones de la seguridad de la tenencia y las condiciones de gobernanza de la tierra (Elementos C y D), que dan como resultado los derechos reales (es decir, los derechos que titulares de derechos practican) (Elemento E). Sostenemos que son sus derechos reales, en lugar de los derechos en la ley, los que incentivan a las personas a tomar decisiones (Elemento F) concernientes a inversiones para la mejora de tierras o de recursos naturales $(\mathrm{G})$.

Con base en la investigación que muestra que las redes sociales internas y de escalas cruzadas es un factor clave que contribuye al éxito de la devolución de los derechos forestales (Baynes et al. 2015), proponemos que las inversiones en capital social (Elemento H) debería considerarse como un tipo de intervención de RPF. Ante la insatisfacción de sus derechos reales, los propietarios de tierras podrían intentar aprovechar su capital social fortalecido (Elemento I) para influir en los sistemas de gobernanza de la tierra (p. ej. mejorar la capacidad de aplicación, ampliar la participación de la comunidad, conciliar políticas inconsistentes) (Flecha 1) de forma que sus derechos reales estén más en línea con sus derechos estatutarios. De maneja alternativa, podrían escoger el uso de su capital social fortalecido para lograr el cambio de la condición de sus derechos estatutarios (Flecha 2) o los derechos estatutarios en sí (Flecha 3), y por lo tanto potencialmente afectando sus derechos reales. En última instancia, las decisiones sobre gestión de tierras y de inversión en capital social que las personas toman en función de sus derechos reales, son las que conducen a resultados socioecológicos (Elemento J), y al éxito de iniciativas en el futuro.

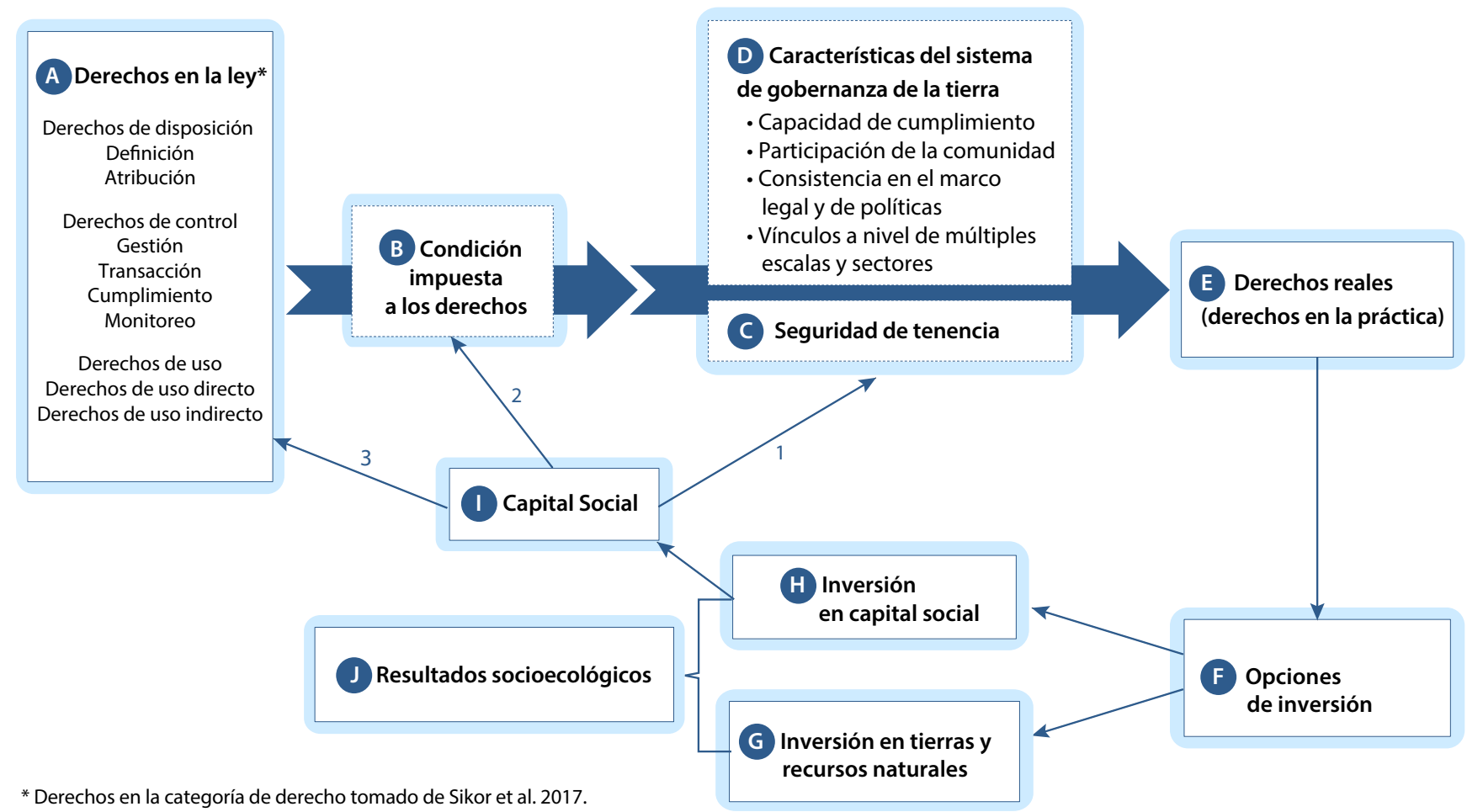

Figura 1. Modelo de actualización de derechos para un diagnóstico de tenencia de oportunidades de restauración. 
Con el fin de poner en operación el modelo de actualización de derechos el análisis sobre tenencia y gobernanza debe responder por lo menos dos preguntas clave:

1. Considerando los mecanismos de tenencia y gobernanza de la tierra en el área, ¿dónde y quiénes es probable que implementen las intervenciones de RPF a gran escala?

2. Considerando los mecanismos de tenencia y gobernanza en el área, ¿dónde y para quiénes habrá impactos negativos de las intervenciones de RPF? Para responder a esta pregunta, deben tenerse particularmente en cuenta los titulares secundarios de derechos, al igual que los titulares principales de derechos en áreas con reclamos superpuestos o donde los derechos a la tierra y al pastoreo, o a la tierra y los árboles, se pueden separar.

Al responder a estas preguntas el análisis debería identificar las características de tenencia y gobernanza de la tierra que afectan probablemente los patrones espaciales y demográficos en la adopción de intervenciones de RPF, así como sus costos y beneficios. Mediante el uso de nuestro modelo de actualización de derechos se podría facilitar el desarrollo de descripciones sistemáticas de los derechos en práctica, así como en los que contempla la ley. Una descripción sólida de los derechos reales debería permitir la identificación de intervenciones de RPF apropiadas a diferentes tipos de derechos. Por ejemplo, probablemente las personas que poseen tierra no estarían interesadas en la plantación de árboles para madera de construcción si la ley forestal no permite a los propietarios cosechar sus árboles. Sin embargo, podrían querer plantar árboles frutales si existe un mercado viable de frutas. Ahora los mismos propietarios de tierra podrían interesarse en unirse en una asociación de usuarios forestales que abogue por reformas legales que otorguen a los propietarios de tierras el derecho a cosechar árboles que hayan sido plantados en sus propiedades.

\section{Recomendaciones para una agenda de RPF sensible al aspecto de tenencia}

También existe la necesidad de un paradigma mejorado en cuanto a derechos para el diseño e implementación de un programa de RPF. A continuación se describen tres elementos críticos para un enfoque de derechos mejorados. Las intervenciones asociadas a abordar todos estos elementos requieren atender explícitamente inequidades sociales y de género respecto al acceso a derechos, beneficios y conectividad social.

Derechos y seguridad de tenencia: Los derechos en combinación con una seguridad de tenencia brindan la base para la RPF con mejora en los derechos. Se requiere abordar dos componentes críticos, el reconocimiento/devolución de derechos y la actualización de derechos.

Reconocimiento/devolución de derechos: En casos en que ya existen derechos consuetudinarios, es recomendable asegurar el reconocimiento formal de estos.

Actualización de los derechos: Estas intervenciones deben concentrarse en facilitar la habilidad de los propietarios de tierras en el ejercicio de sus derechos estatutarios y en reducir las condiciones en los derechos que producen desincentivos relacionados con la tenencia para la restauración. Los mecanismos para mejorar la actualización de los derechos incluyen programas que mejoran la rendición de cuentas dentro de los sistemas de gobernanza de la tierra, proporcionan procesos de resolución de conflictos accesibles y asequibles, mejoran las competencias judiciales y crean capacidades de aplicación y gestión a nivel local.

Mejorar la conectividad entre escalas, sectores y actores sociales: Los informes ROAM identifican las brechas en la conectividad entre y dentro de las escalas de gobernabilidad, así como entre los sectores y los diferentes tipos de actores sociales, como obstáculos para la implementación de la RPF. Un área focal clave para las mejoras de la conectividad social es el apoyo a las redes de grupos de usuarios basadas en la comunidad con fuertes enlaces internos y externos con otras partes interesadas. Las plataformas de múltiples partes interesadas han demostrado ser útiles para construir y fortalecer los vínculos entre los actores dentro y entre las escalas de gobierno y los diferentes sectores (Pinto et al. 2014). Brouwer et al. (2016) idearon un conjunto de principios clave para construir alianzas efectivas entre múltiples partes interesadas, incluyendo el cambio sistémico, la transformación de las instituciones, el trabajo con poder, el manejo del conflicto, la promoción del liderazgo colaborativo, el fomento del aprendizaje participativo y la comunicación efectiva.

Diseños de programas que generan beneficios ampliamente compartidos: la distribución desigual de beneficios surgió como una preocupación en muchos de los informes ROAM. Las inequidades en la distribución de beneficios también se encuentran en muchos de los proyectos REDD+ a pesar de las salvaguardas sociales, con una tendencia a dejar fuera a poblaciones indígenas, mujeres y los hogares más pobres (Larson et al. 2015; Ickowitz et al. 2017; Sarmiento Barletti y Larson 2017). Las iniciativas de RPF que caen fuera de la cobertura de REDD+ tendrán mayores costos de oportunidad para la agricultura o pueden tener un impacto negativo en el acceso a los recursos por parte de los titulares secundarios de derechos. El brindar fuertes beneficios forestales mediante la devolución de derechos de cosecha de árboles y productos comerciales para uso comercial, puede ayudar a compensar los altos costos de oportunidad de los bosques en relación con usos agrícola de la tierra.

\section{Conclusión}

El manual ROAM y el Diagnóstico de Restauración identifican los elementos clave necesarios para hacer un análisis de tenencia y gobernanza de la tierra. Sin embargo, no proporcionan un marco que muestre cómo esos elementos están conectados entre sí o con tipos específicos de intervenciones de RPF. Al posibilitar a los planificadores el mejor entendimiento de dichas conexiones, nuestro modelo debe apoyar el diseño de programas de RPF a la medida del contexto de tenencia y gobernanza de la tierra en el cual se implementan. La cobertura en temas de tenencia y gobernanza de la tierra en los informes ROAM puede estar relacionada también con la composición de los equipos evaluadores, los cuales parecen estar conformados principalmente por personas con experiencia en el campo de las ciencias naturales. Una solución posible es incluir en el equipo principal por lo menos un experto en tenencia de tierras con experiencia como investigador, o entrenar en la realización de análisis de tenencia y gobernanza a miembros del equipo principal. 
Como con cualquier otro modelo, el nuestro es una simplificación de la realidad. Los elementos y relaciones que hemos descrito son influidos por otros factores como los costos de oportunidad de la inversión en la RPF, el estatus social y de riqueza, y la distancia a la cual se encuentran los mercados de productos forestales, entre otros. La forma en que estos factores interactúan con los elementos del modelo, cómo y cuándo los propietarios de tierra o los usuarios de tierra construyen conexiones sociales y las activan para influir en la gobernanza de la tierra y las dimensiones de la tenencia, y cuáles son los resultados de la activación de las conexiones sociales sobre los derechos reales y las opciones de inversión, son áreas que necesitan ser investigadas adicionalmente. Un paso lógico subsiguiente consiste en probar el modelo para ver qué tan bien funciona en la práctica a escalas nacional y subnacional.

El modelo de actualización de derechos que proponemos es aplicable más allá de la metodología ROAM o de otras evaluaciones de restauración forestal. Los principios generales deben ser válidos para cualquier contexto de planificación en el que estén en juego los derechos de tenencia y la seguridad. Por lo tanto, es igualmente útil como una herramienta potencial en otros sectores de recursos naturales, como la gestión de bosques, desarrollo agrícola y la gestión de la pesca, donde los planificadores tratan de producir un cambio en el comportamiento de la gestión de la tierra y los recursos.

\section{Agradecimientos}

El apoyo a este trabajo se obtuvo mediante recursos financieros del Programa de Investigación sobre Políticas, Instituciones y Mercados (PIM) del CGIAR. Los autores expresan su agradecimiento a Himal Baral y Ruth Meizen-Dick por sus comentarios en los primeros borradores de este Infobrief.

Este Infobrief es una adaptación y una condensación de la publicación: Rebecca McLain, Steven Lawry, Manuel R. Guariguata y James Reed. 2018. Toward a tenure-responsive approach to forest landscape restoration: A proposed tenure diagnostic for assessing restoration opportunities. Land Use Policy. https://doi.org/10.1016/j. landusepol.2018.11.053

\section{Referencias}

Baynes J, Herbohn J, Smith C, Fisher R y Bray D. 2015. Key factors which influence the success of community forestry in developing countries. Global Environmental Change Part A 35:226-38.

Brouwer H, Woodhill J, Hemmati M, Verhoosel K y van Vugt S. 2016. The MSP Guide, How to Design and Facilitate Multi-stakeholder Partnerships. Wageningen University and Research, CDI, y Rugby, Reino Unido: Practical Action Publishing, Wageningen. Consultado el 10 de septiembre de 2018. https://doi. org/10.3362/9781780446691

Cronkleton P, Artati Y, Baral H, Paudyal K, Banjane MR, Liu JJ, Tu TY, Putzel L, Birhane E y Kassa H. 2017. How do property rights reforms provide incentives for forest landscape restoration? Comparing evidence from Nepal, China and Ethiopia. International Forestry Review 19(54):8-23.
Ickowitz, A Sills, E y de Sassi C. 2017. Estimating smallholder opportunity costs of REDD+: a pantropical analysis from households to carbon and back. World Development 95:15-26.

Larson AM, Dokken T, Duchelle AE, Atmadja S, Resosudarmo IAP, Cronkleton P y Selaya G. 2015. The role of women in early REDD+ implementation: Lessons for future engagement. International Forestry Review 17(1):43-65.

Lawry S, Samii C, Hall R, Leopold A, Hornby D y Mtero F. 2016. The impact of land property rights interventions on investment and agricultural productivity in developing countries: A systematic review. Journal of Development Effectiveness 1:1-21.

Mansourian S. 2017. Governance and forest landscape restoration: A framework to support decision-making. Journal for Nature Conservation 37:21-30.

Pinto SR, Melo F, Tabarelli M, Padovesi A, Mesquita CA, de Mattos Scaramuzza CA, Castro P, Carrascosa H, Calmon M, Rodrigues R, César RG y Brancalion PHS. 2014. Governing and delivering a biome-wide restoration initiative: The case of Atlantic Forest Restoration Pact in Brazil. Forests 5(9):2212-29.

Sarmiento Barletti JP y Larson A. 2017. Rights abuse allegations and REDD+: A review and proposal for moving forward. CIFOR Infobrief no. 190. Bogor, Indonesia: Centro para la Investigación Forestal Internacional (CIFOR). https://doi.org/10.17528/ cifor/006630

Sikor T, He J y Lestrelin G. 2017. Property rights regimes and natural resources: A conceptual analysis revisited. World Development 93:337-49.

Stanturf J, Mansourian S y Kleine M, eds. 2017. Implementing Forest Landscape Restoration, a Practitioner's Guide. Viena, Austria: Unión Internacional de Organizaciones de Investigación Forestal.

[UICN] Unión Internacional para la Conservación de la Naturaleza. 2019. Restoration Opportunities Assessment Methodology (ROAM). UICN. Consultado el 16 de enero de 2019. www.iucn.org/ theme/forests/our-work/forest-landscape-restoration/restorationopportunities-assessment-methodology-roam

[UICN] Unión Internacional para la Conservación de la Naturaleza. 2018. InfoFLR. UICN. Accessed 16 January 2019. https://infoflr.org/

[UICN] Unión Internacional para la Conservación de la Naturaleza. s.f. Bonn Challenge. Washington, DC: UICN. Consultado el 16 de enero de 2019. http://www.bonnchallenge.org/

[UICN/WRI] Unión Internacional para la Conservación de la Naturaleza/Instituto de Recursos Mundiales. 2014. A guide to the Restoration Opportunities Assessment Methodology (ROAM): Assessing forest landscape restoration opportunities at the national or sub-national level. Working Paper (Road-test edition). Gland, Suiza: UICN.

von Benda-Beckmann F. y von Benda-Beckmann K. 2000. Gender and the multiple contingencies of water rights in Nepal. En: Pradhan R, von Benda-Beckmann F y von Benda-Beckmann K, eds. Water, Land and Law: Changing Rights to Land and Water in Nepal. Kathmandu/Wageningen/Rotterdam: Legal Research and Development Forum (FREEDEAL)/ Universidad Agrícola de Wageningen (WAU)/Universidad Erasmus de Rotterdam (EUR) 17-38.

[WRI] Instituto de Recursos Mundiales. 2015. The Restoration Diagnostic: A Method for Developing Forest Landscape Restoration Strategies by Rapidly Assessing the Status of Key Success Factors. Washington, DC: Instituto de Recursos Mundiales. 


\section{Apéndice}

\section{Informes de Metodología de Evaluación de Oportunidades de Restauración}

[CERGIS/WRI/UICN] Centro de Teledetección y Servicios de Información Geográfica/Instituto de Recursos Mundiales/Unión Internacional para la Conservación de la Naturaleza y Universidad Estatal de Dakota del Sur. 2011. Assessment of Forest Landscape Restoration Opportunities in Ghana. Final Report. PROFOR. Sin fecha de publicación. Consultado el 9 de septiembre de 2018. https://www.profor.info/sites/profor.info/files/Ghana\%20FLR\%20 PROFOR\%20final\%20report\%2029\%20September_0.pdf

Diederichsen A, Gatti G, Nunes S, y Pinot A. 2017. Diagnóstico dos Fatores Chave de Sucesso para a Restauração da Paisagem Florestal. Município de Paragominas e Estado do Pará Componente da Metodologia de Avaliação de Oportunidades de Restauração (ROAM). Belém, PA: Instituo do Homem e Meio Ambiente da Amazônia (Imazon); Curitiba: Conserve Brasil. Consultado el 17 de enero de 2019.

Instituto Nacional de Bosques, Programa Forestal Nacional y Unión Internacional para la Conservación de la Naturaleza. 2014. Análisis del marco normativo y regulatorio, mapeo de iniciativas y diagnóstico de la situación de la restauración del paisaje forestal en Guatemala. Elaborado en comisión para: Mesa Nacional de Restauración del Paisaje Forestal: Guatemala. Documento sin publicar. En archivo con el autor.

[UICN] Unión Internacional para la Conservación de la Naturaleza. 2016. Opportunités de restauration des forêts et paysages dégradés en Côte d'Ivoire. Rapport Final. Ouagadougou, Burkina Faso: UICN. Consultado el 9 de septiembre de 2018. https://www.iucn.org/ sites/dev/files/content/documents/opportunites_de_restauration_ des_forets_et_paysages_degrades_en_cote_divoire.pdf
Nunes S, Gatti G, Diederichsen A, Silva D y Pinto A. 2017. Oportunidades para restauração florestal no Estado do Pará. Belém, Brazil: Instituo do Homem e Meio Ambiente da Amazônia (Imazon). Consultado el 9 de septiembre de 2018.

https://imazon.org.br/PDFimazon/Portugues/livros/Oportunidade\%20 Restauracao\%20Florestal\%20Para.pdf

República de Malawi, Ministerio de Recursos Naturales, Energía y Minas. 2017. Forest landscape restoration opportunities assessment for Malawi. National Forest Landscape Restoration Assessment (Malawi). Blantyre, Malawi: Centro para Política y Defensa Ambiental (CEPA). Consultado el 9 de septiembre de 2018. https://cepa.rmportal.net/Library/inbox/ forest-landscape-restoration-opportunities-assessment-for-malawi/ view

República de Ruanda, Ministerio de Recursos Naturales. 2014. Forest Landscape Restoration Opportunity Assessment for Rwanda. Nairobi, Kenia: MINIRENA (Ruanda), Unión Internacional para la Conservación de la Naturaleza (UICN), Instituto de Recursos Mundiales (WRI). Consultado el 9 de septiembre de 2018. https://www.iucn.org/ content/forest-landscape-restoration-opportunity-assessment-rwanda República de Uganda, Ministerio de Agua y Medio Ambiente y [UICN] Unión Internacional para la Conservación de la Naturaleza. 2016. Forest Landscape Restoration Opportunity Assessment for Uganda. Kampala, Uganda: Ministry of Water and Environment. Consultado el 9 de septiembre de 2018. https://www.iucn.org/content/forestlandscape-restoration-opportunity-assessment-uganda

Silva, D y Nunes, S. 2017. Avaliação e modelagem econômica da restauração florestal no Estado do Pará. Belém, Brazil: Instituo do Homem e Meio Ambiente da Amazônia (Imazon). Consultado el 9 de septiembre de 2018. http://imazon.org.br/publicacoes/avaliacao-emodelagem-economica-da-restauracao-florestal-no-estado-do-para/

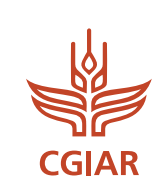

RESEARCH PROGRAM ON Policies Institutions, and Markets Led by IFPRI
El Programa de Investigación sobre Políticas, Instituciones y Mercados (PIM) del CGIAR, lidera investigación orientada a la acción para equipar a tomadores de decisiones con los resultados requeridos para desarrollar políticas alimentarias y agrícolas que satisfagan mejor los intereses de productores pobres y de consumidores, tanto hombres como mujeres. El PIM combina los recursos de los centros CGIAR y varios socios internacionales, regionales y nacionales. El programa es liderado por el Instituto de Investigación sobre Políticas Alimentarias (IFPRI). www.pim.cgiar.org desarrollando las capacidades de sus socios y dialogando activamente con todos los actores involucrados, para informar sobre las políticas y las prácticas que afectan a los bosques y a las personas. CIFOR es un centro de investigación CGIAR y lidera 